\title{
Late sequelae of paralytic poliomyelitis: a clinical and electromyographic study
}

\author{
M I C H A E L H A Y A R D A D DOUGLAS SEATON \\ From the Regional Neurological Unit, Walton Hospital, Liverpool
}

SUMMARY A prospective study was carried out on 24 volunteers who had suffered from paralytic poliomyelitis up to 51 years earlier. A quantitative EMG was carried out in each subject. Grossly raised mean amplitudes of the interference patterns were found in many strong muscles as well as in weak muscles. Such muscles frequently showed "contraction fasciculation", a manifestation of loss of normal small motor units. In many subjects the clinical and EMG evidence of chronic partial denervation were more widespread than the subjects had realised. It is suggested that if, with advancing age, minor damage occurs to the peripheral nervous system and the complement of motor neurones is already depleted by poliomyelitis, there will be an exaggerated response with increase in the lower motor neurone signs.

Late deterioration of function is a well-documented sequel in a proportion of patients who have suffered from paralytic poliomyelitis in earlier days (Anderson et al., 1972). There have been a number of reports describing the development of motor neurone disease in such patients (KayserGatchalian, 1973). In our experience of patients referred to us with this diagnosis there has in each case been a more acceptable explanation for the deterioration, and the resemblance to motor neurone disease has been superficial. This experience prompted us to undertake a prospective clinical and electromyographic (EMG) study of a group of 24 subjects, not currently attending hospital, who had suffered from paralytic poliomyelitis up to 51 years earlier, paying particular attention to the manifestations and extent of chronic partial denervation and to evidence of deterioration of function.

\section{Subjects and methods}

The subjects were 15 men and nine women volunteers who were identified with the co-operation of a sports society for the disabled and through the diagnostic index of a local infectious diseases unit. A further two subjects who were interviewed were rejected from the study because of doubt as to

Address for reprint requests: Dr M. Hayward, Department of Medical Neurology, Walton Hospital, Rice Lane, Liverpool L9 1AE.

Accepted 12 September 1978 the true nature of their original illness. All the subjects were questioned in detail about their functional abilities and underwent a thorough physical examination which included measurement of the maximum voluntary effort of major muscle groups with a spring balance and measurement of the length and girth of the limbs. In each subject an objective measure of chronic partial denervation was made on one or more muscles by carrying out a quantitative EMG against standard load. The load on the muscle during the EMG examination was controlled using a strain gauge transducer whose output was amplified and displayed to the subject and the examiner on a large scale analogue meter. The examination technique and method of analysis were otherwise as described in detail by Hayward (1977). The bandwidth of the amplifiers used (Medelec Ltd) was set from $8 \mathrm{~Hz}-$ $16 \mathrm{kHz}$.

\section{Results}

The results of the quantitative EMG and details of the degree of clinical disability of the 24 subjects are listed in the Table. The subjects have been grouped according to the muscle chosen for EMG examination and each group arranged in order of the time elapsed since the acute poliomyelitis infection. The ages of the subjects ranged from 1868 years and their poliomyelitis had occurred 13-51 years earlier. The degree of residual dis- 
Table Subjects with old poliomyelitis listing the extent of the initial paralysis, degree of residual disability (see text), maximum voluntary effort (MVE) of muscles examined by EMG and results of quantitative EMG carried out at fixed load (abnormal values in bold type)

\begin{tabular}{|c|c|c|c|c|c|c|c|c|c|c|c|}
\hline $\begin{array}{l}\text { Series } \\
\text { number }\end{array}$ & Sex & $\begin{array}{l}\text { Age } \\
\text { (yr) }\end{array}$ & $\begin{array}{l}\text { Age at } \\
\text { onset } \\
(y r)\end{array}$ & $\begin{array}{l}\text { Duration } \\
(y r)\end{array}$ & Initial paralysis & $\begin{array}{l}\text { Disability } \\
\text { scale }\end{array}$ & & Muscle examined & $\begin{array}{l}M V E \\
(k g)\end{array}$ & $\begin{array}{l}\text { Mean } \\
\text { turns } \\
\text { (per second) }\end{array}$ & $\begin{array}{l}\text { Mean } \\
\text { amplitude } \\
(m V)\end{array}$ \\
\hline 1 & $\mathbf{M}$ & 40 & 23 & 17 & Tetraparesis & 3 & & Right biceps brachii & 15 & 200 & 0.80 \\
\hline 2 & $\mathbf{M}$ & 19 & 1 & 18 & Paraparesis & 2 & & Left biceps brachii & 23 & 440 & 0.47 \\
\hline \multirow[t]{2}{*}{3} & $\mathbf{M}$ & 21 & 2 & 19 & Right arm & 1 & a) & Right biceps brachii & 20 & 161 & 07.1 \\
\hline & & & & & & & b) & Left biceps brachii & 30 & 321 & 0.36 \\
\hline 4 & $\mathbf{M}$ & 50 & 23 & 27 & Tetraparesis & 3 & & Right biceps brachii & 18 & 101 & 0.62 \\
\hline 5 & $\mathbf{F}$ & 46 & 14 & 32 & Both legs, left arm & 3 & & Left biceps brachii & 18 & 206 & 0.69 \\
\hline 6 & $\mathbf{M}$ & 56 & 10 & 46 & Paraparesis & 3 & & Left biceps brachii & 14 & 264 & $\mathbf{0 . 5 5}$ \\
\hline 7 & $\mathbf{M}$ & 55 & 4 & 51 & Tetraparesis & 2 & & Left biceps brachii & 15 & 230 & 1.29 \\
\hline 8 & $\mathbf{M}$ & 44 & 31 & 13 & Both arms, left leg & 1 & & Right vastus medialis & 21 & 163 & 1.00 \\
\hline 9 & $\mathbf{F}$ & 18 & 3 & 15 & Right leg & 0 & & Right vastus medialis & 25 & 218 & 1.22 \\
\hline \multirow[t]{2}{*}{10} & $\mathbf{M}$ & 21 & 6 & 15 & Right leg & 0 & a) & Right vastus medialis & 32 & 206 & 0.96 \\
\hline & & & & & & & b) & Left vastus medialis & 33 & 142 & 0.51 \\
\hline 11 & $\mathbf{F}$ & 43 & 26 & 17 & Right leg & 1 & & Right vastus medialis & 20 & 355 & 1.19 \\
\hline \multirow[t]{2}{*}{12} & $\mathbf{M}$ & 22 & 5 & 17 & Left leg & 0 & a) & Right vastus medialis & 32 & 101 & 0.48 \\
\hline & & & & & & & b) & Left vastus medialis & 32 & 125 & 0.78 \\
\hline 13 & $\mathbf{M}$ & 29 & 10 & 19 & Tetraparesis & $\mathbf{0}$ & & Right vastus medialis & 35 & 76 & 0.71 \\
\hline 14 & $\mathbf{F}$ & 24 & 5 & 19 & Right arm, right leg & 0 & & Right vastus medialis & 25 & 114 & 0.26 \\
\hline 15 & $\mathbf{M}$ & 27 & 2 & 20 & Left arm, left leg & 0 & & Left vastus medialis & 35 & 168 & 0.79 \\
\hline 16 & $\mathbf{M}$ & 21 & 1 & 20 & Left leg & 1 & & Left vastus medialis & 20 & 184 & 1.08 \\
\hline 17 & $\mathbf{M}$ & 21 & 1 & 20 & Right leg & 1 & & Right vastus medialis & 28 & 128 & 0.65 \\
\hline 18 & $\mathbf{F}$ & 24 & 1 & 23 & Tetraparesis & 1 & & Right vastus medialis & 14 & 216 & 0.91 \\
\hline 19 & $\mathbf{F}$ & 27 & 2 & 25 & Paraparesis & 1 & & Left vastus medialis & 8 & 300 & 0.96 \\
\hline 20 & $\mathbf{M}$ & 44 & 2 & 42 & Left leg & 0 & & Left vastus medialis & 29 & 247 & 0.39 \\
\hline 21 & $\mathbf{F}$ & 68 & 23 & 45 & Right arm, right leg & 2 & & Left vastus medialis & 25 & 144 & 0.35 \\
\hline 22 & $\mathbf{M}$ & 42 & 29 & 13 & Paraparesis & 2 & & Left tibialis anterior & 4 & 98 & 0.19 \\
\hline 23 & $\mathbf{F}$ & 24 & 10 & 14 & Tetraparesis & 2 & & Left tibialis anterior & 20 & 330 & 1.47 \\
\hline 24 & $\mathbf{F}$ & 18 & 2 & 16 & Left thigh & 0 & & Left tibialis anterior & 20 & 412 & 0.49 \\
\hline
\end{tabular}

Normal mean values (Hayward, 1977): biceps brachii, mean turns 371/s, SD 89; mean amplitude $0.35 \mathrm{mV}$, SD 0.07 ; vastus medialis, mean turns 263/s, SD 73; mean amplitude $0.33 \mathrm{mV}$, SD 0.05; tibialis anterior, mean turns 307/s, SD 43; mean amplitude $0.45 \mathrm{mV}, \mathrm{SD} 0.09$.

ability was graded $0-3$. The disability of eight subjects was graded 0 -that is, they were completely free of any disabling symptoms. Grade 1 disability (seven subjects), indicated the presence of mild symptoms, not sufficiently severe as to impair mobility in the home, or the ability to use public transport or to work in the home or in employment. Several of these seven subjects were employed in physically strenuous jobs such as labouring on the docks. None used any form of physical appliance although many had used calipers or other appliances for several years after the acute attack. Grade 2 disability (five subjects) indicated some loss of mobility, inability to use normal public transport, the necessity for sheltered employment, or the continuing use of a physical appliance to aid mobility. The four subjects with grade 3 disability were severely handicapped and confined to wheelchairs. Subject 5 had become a champion archer despite having flail legs and initial involvement of the left arm. Subject 1 was the most severely disabled with flail legs and moderately severe involvement of both arms. Ten of the subjects from all the disability grades had had orthopaedic operations carried out to improve function and reduce deformity. Opera- tions included tenotomies, tendon transpositions, and one spinal fusion. Several subjects had undergone multiple operations.

Many of the subjects of all grades of disability had relatively minor symptoms of long duration that they regarded as part of their heritage. The most common of these symptoms was cramp, which affected both weak muscles and some which were relatively strong. In general those subjects with grades 2 and 3 disability had tended to become less mobile as they had grown older. Subjects 6, 19, and 23 had lost their employment because of their decreasing mobility. A number of subjects had sustained fractures when falling because of weakness or instability of a limb. Six subjects complained of joint pains, usually associated with local weakness or instability, which had tended to become more troublesome with increasing age. Subject 1 , who had spent the first seven weeks of his acute illness in a respirator and had been in hospital for more than a year, complained that as he had become older he had suffered from respiratory infections with increasing frequency. He had significant residual weakness of the intercostal muscles as well as of arms and legs. The findings on physical examination of those sub- 
jects who admitted to decreasing mobility differed in no way from those other subjects with the same grade of disability whose function was not obviously deteriorating.

The most common physical sign of old poliomyelitis was reduction of muscle bulk. When the subject had been affected during infancy this was associated with shortening of the limb. Muscle power was reduced in all the muscles that were severely wasted but was often surprisingly well preserved in muscles that were only moderately reduced in bulk.

Most subjects showed "contraction fasciculation" (Denny-Brown and Pennybacker, 1938) in the overtly affected muscles and also quite frequently in muscles that were clinically otherwise normal or only slightly reduced in bulk and strength. If the muscles were not completely relaxed, or during slight deliberate contraction, regular contraction of muscle fascicles could be seen under the skin and could be felt by palpating the muscle. As the degree of voluntary contraction was increased the twitching became more prominent, making the surface of the muscle appear to ripple beneath the skin. The motor unit action potentials that were identified with the clinical phenomenon of contraction fasciculation were usually of relatively high amplitude. These motor units could be shown to be under the influence of normal control mechanisms. Their discharge frequencies increased when the subject was asked to increase gently contraction in the muscle, and their activity always ceased with careful positioning of the subject's limb. No attempt was made to estimate the twitch tensions of these units but it was frequently observed during the EMG examinations that the subjects could not maintain a muscle contraction of moderate force as evenly as a normal individual. Examination of the output of the strain gauge showed a coarse ripple superimposed on the basic contraction. This feature was not confined to weak muscles. It was always associated with contraction fasciculation and both features were invariably associated with abnormalities of the EMG in that muscle (increase in mean amplitude of the interference pattern even when the muscle had normal bulk and strength.

Spontaneous fasciculation-that is, irregular contraction of motor units dissociated from voluntary effort-was observed in only five subjects and was never profuse. Another two subjects stated that they were occasionally aware of spontaneous twitching although this was not identified during their physical examination. Repetitive high frequency discharges were recorded from two areas of muscle in subject 22 , a very severely affected muscle whose EMG features were not typical of the majority. Spontaneous fibrillation was not recorded.

When selecting muscles on which to carry out quantitative EMG examinations we were not interested in examining those which were severely weak and wasted but rather to study muscles which were either normal on clinical examination, whether or not there was a history of the muscle being weak during the acute attack, or which were moderately weak or showed contraction fasciculation. Muscles that might have been affected by tenotomies or other forms of operative treatment were rejected. In the leg vastus medialis and tibialis anterior were studied; in the arm biceps brachii was studied.

In muscles with reduced bulk and power the mean amplitude of the interference pattern recorded at standard load was grossly elevated, with the single exception of subject 22 , whose tibialis anterior was the weakest of all the muscles examined (the finding of myotonia in this muscle may have indicated an acute disturbance of function superimposed on the chronic disease). The results are listed in the Table. Significant increase in mean amplitude-2 SD or more above the control group mean-was also found many times in muscles of normal bulk and power. This was particularly striking in vastus medialis, the muscle most frequently studied. The results of the examinations of vastus medialis are illustrated in Fig. 1 where they are compared with the results from patients with motor neurone disease (Hayward and Willison, 1977) and with the results from healthy subjects (Hayward, 1977). Some of the muscles with interference pattern of increased mean amplitude appeared to be entirely normal on clinical examination alone.

In any individual muscle with an increased mean amplitude of interference pattern the scatter of the amplitudes of the 15 or more areas that were examined in that muscle was similar to that found in cases of chronic partial denervation from any other cause. Figure 2 illustrates an example of this, subject 10 , a young man of 21 years who had suffered from paralytic polio myelitis 15 years earlier. His original case records commented that his right leg had been paralysed but made no mention of weakness of the left leg. When examined for this study no shortening of the leg was noted but the right thigh was $75 \mathrm{~mm}$ thinner than the left. Maximum voluntary effort of knee extension was not greatly different on the two sides, right $32 \mathrm{~kg}$, left $33 \mathrm{~kg}$. On the right side the mean EMG amplitude in vastus medialis was $0.96 \mathrm{mV}, 12 \mathrm{SD}$ above the control group mean 


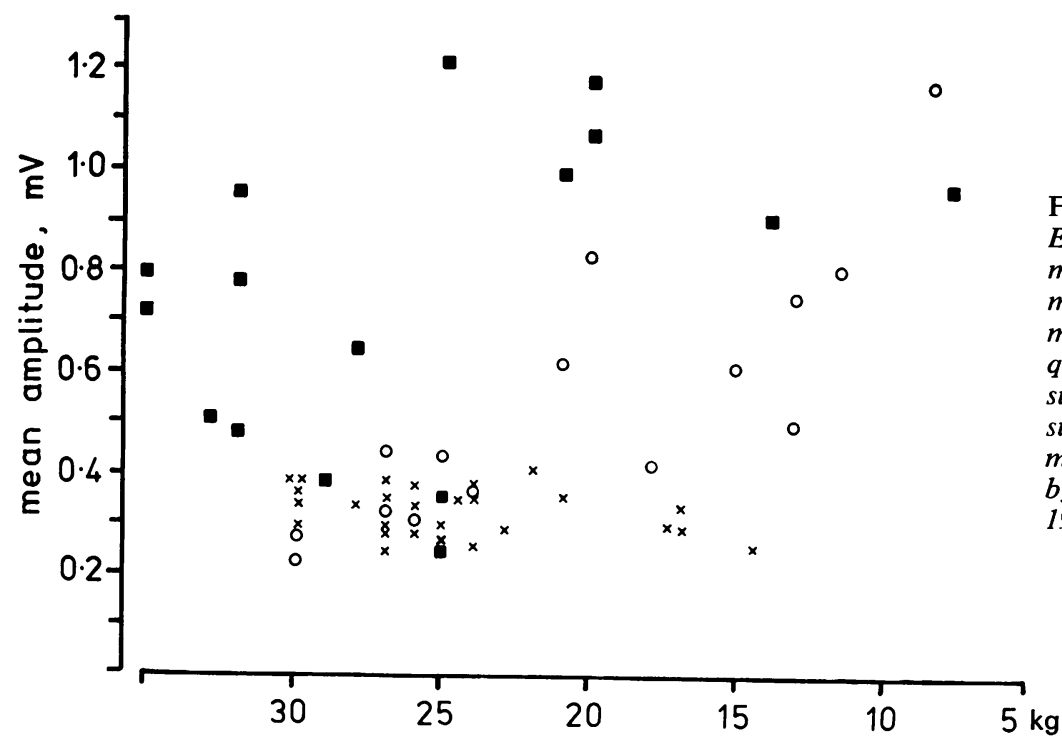

Fig. 1 Results of quantitative EMG examinations of vastus medialis, recorded at $5 \mathrm{~kg}$ load; mean amplitude plotted against maximum voluntary effort of quadriceps. Poliomyelitis subjects indicated by $\mathbf{\square}$, healthy subjects by $\times$ (Hayward, 1977), motor neurone disease patients by $\bigcirc$ (Hayward and Willison, 1977).

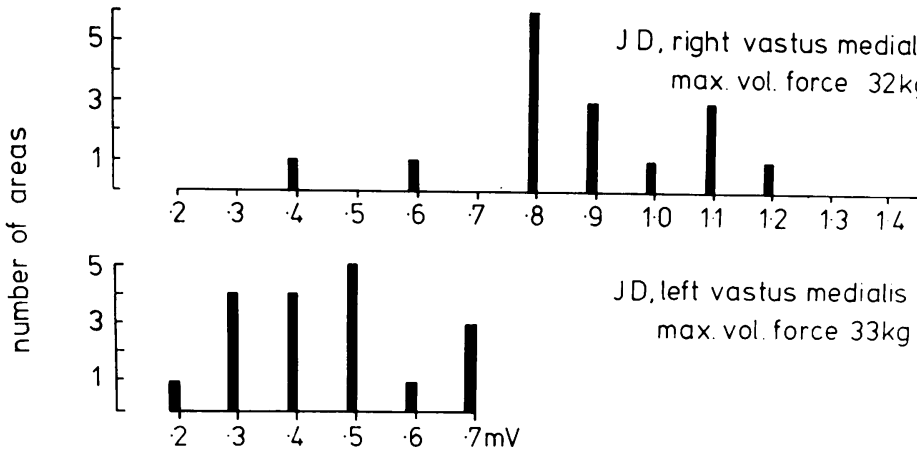

Fig. 2 Histograms of mean amplitudes of individual areas of muscle, right and left vastus medialis, from poliomyelitis subject 10. Comparable data from control series below. All examinations at $5 \mathrm{~kg}$ load.

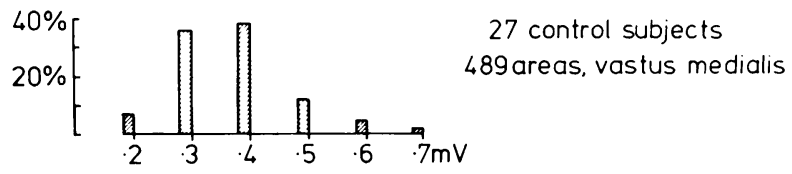

(Hayward, 1977). On the clinically unaffected left side the elevation of mean EMG amplitude was much less striking $(0.51 \mathrm{mV})$ but still highly significant being 3.6 SD above the control group mean. Contraction fasciculation was noted in the right vastus but not in the left. The distributions of the mean EMG amplitudes of the individual areas examined in the two muscles are illustrated as histograms in Fig. 2 together with comparable data from the control series (Hayward, 1977).
In all the muscles examined the turns counts (Hayward, 1977) were within the control group range. There was a tendency for the turn counts of individual subjects of the poliomyelitis series to be lower than the mean values of the control series.

\section{Discussion}

The prime aim of this study was to document by 
clinical and EMG means the extent and degree of chronic partial denervation in a group of subjects who had previously suffered from paralytic poliomyelitis, and to seek evidence of reasons for possible late deterioration of function. We found in most of our subjects that there was both clinical and EMG evidence of denervation that was far more extensive than the subjects realised. Electromyographic evidence of chronic partial denervation was found many times in muscles that were of normal strength and was not confined to the weak and wasted muscles. After wasting, the next most informative physical sign of chronic partial denervation was contraction fasciculation which was, in every case examined, associated with EMG signs of denervation in that muscle. Spontaneous fasciculation was relatively uncommon in these subjects.

A clear distinction between contraction fasciculation and spontaneous fasciculation was drawn by Denny-Brown and Pennybacker (1938) but this distinction has tended to be blurred by a number of subsequent authors. Although both phenomena are features of chronic partial denervation their origins and their significance differ, and we believe that there is every reason to continue to make a clear distinction between them. We observed that the motor units associated with contraction fasciculation were under the control of normal voluntary control mechanisms, and they appeared to be recruited into the interference pattern in a normal way, having action potentials differing from normal only in their high amplitudes. It is a common observation, that we have confirmed on many occasions, that motor units showing spontaneous fasciculation can not be recruited into the interference pattern with voluntary activity.

The site of origin of spontaneous fasciculation has been shown to be distal by the experiments of Forster and Alpers (1944) and of Forster et al. (1946). Personal observations on patients with motor neurone disease confirm that paralysis of voluntary motor function with local anaesthetic nerve block does not reduce the frequency of spontaneous fasciculation, whose occurrence is possibly a manifestation of instability of cell membranes in the distal sprouting axons. In contrast to spontaneous fasciculation, contraction fasciculation is a central phenomenon which we interpret as being caused by the replacement of the small motor units that are normally first recruited during weak effort (Milner-Brown et al., 1973) by greatly enlarged and compacted motor units. These enlarged motor units are visible beneath the skin as they are recruited during initial contraction, producing the clinical phenomenon of contraction fasciculation. Our evidence for this interpretation is the constant association of contraction fasciculation with increase in the mean amplitude of the EMG and the observed ability of the subjects to control the firing rates of the motor units associated with the sign.

A preponderance of high amplitude motor unit potentials has been regarded as characteristic of poliomyelitis since the earliest days of clinical electromyography (Buchthal and Høncke, 1944). The technique of quantitative electromyography that we have used to study these subjects has allowed the degree of abnormality to be documented. Hayward and Willison (1977) have shown that increase in the mean amplitude of the interference pattern recorded at standard load is a reliable index of chronic partial denervation and have argued that the increase is a manifestation of reinnervation in a partially denervated muscle. They studied patients with motor neurone disease and in that condition found that mean amplitude increased as the disease progressed. In motor neurone disease increased mean EMG amplitudes were never found in muscles of normal strength, the degree of increase being related to the strength of the muscle. The EMG results from the present series of subjects with old poliomyelitis contrast with the results from the motor neurone disease patients in that very high mean EMG amplitudes were found in muscles of normal strength in some of the poliomyelitis subjects (Fig. 1). A muscle with unit potentials of grossly increased mean amplitude must have a severely depleted complement of motor units, the preserved strength (and bulk) indicating that the majority of muscle fibres have been successfully reinnervated or replaced in such muscles.

Poliomyelitis contrasts with motor neurone disease in that it is an acute illness in which a substantial recovery can take place in muscles that are not too severely damaged, where a sufficient number of anterior horn cells survive. The degree of reinnervation that can occur will depend upon the number of surviving anterior horn cells but also on the age of the subject at the time of the infection. Many aspects of repair and of response to injury of the nervous system become less efficient with advancing age (Gutmann and Hanzlikova, 1972). The average age at which the present subjects suffered from poliomyelitis was 31.4 years while that of the motor neurone disease patients reported by Hayward and Willison was 53 years at the time of their presentation. Additionally, the progressive nature of motor neurone disease will limit the degree to which reinnervation can occur. 
The slight deterioration of function, often associated with cramps and local joint pains, that we found among the more severely affected of the group can be explained largely on the basis of a gradual reduction of physical fitness, whatever its cause, that is common to most individuals with advancing age. There may, however, be more specific causes for deterioration in some cases. Hayward (1977) has produced evidence that subclinical denervation may be found in muscles of "healthy" individuals with advancing age. The reasons for this finding are still far from certain but on the basis that denervation was not found in all the muscle groups studied he postulated that "ageing" was not the sole factor involved, and that perhaps local factors, such as repeated minor trauma to nerve roots or to peripheral nerves, might be relevant, the subjects being less able to repair damage to the nervous system with increasing age. This study has shown that in the poliomyelitis subjects even strong muscles may show signs of significant denervation-that is, they are functioning with a reduced complement of motor units, each of which must contribute a greater proportion of the strength of the muscle than in the healthy situation. The poliomyelitis subjects will be less able to compensate for any further loss of motor units with advancing age without producing new clinical signs. If reinnervation through the peripheral sprouting of axons does occur then the surviving motor units will be even further enlarged and contraction fasciculation will become prominent. If sprouting is unsuccessful then an affected muscle that was previously strong will start to show weakness. It seems very likely that from time to time in poliomyelitis subjects local deterioration of function will occur in muscles controlled by a depleted number of motor neurones with apparent increase in the lower motor neurone signs. When this occurs the diagnosis may be confused with motor neurone disease by an inexperienced observer, particularly if contraction fasciculation and spontaneous fasciculation are not clearly distinguished. We found that our subjects were not always reliable witnesses as to the extent of their previous poliomyelitis being unaware of the subclinical involvement of many muscles.

We are grateful to $\mathrm{Mr} \mathrm{W}$. A. L. Thompson for his help in locating subjects, to Dr I. R. Williams for his helpful comments on the manuscript, and to the subjects themselves for their ready cooperation in this study.

\section{References}

Anderson, A. D., Levine, S. A., and Gellert, H. (1972). Loss of ambulatory ability in patients with old anterior poliomyelitis. Lancet, 2, 1061-1063.

Buchthal, F., and Høncke, P. (1944). Electromyographical examination of patients suffering from poliomyelitis ant. ac. up to 6 months after the acute stage of the disease. Acta Medica Scandinavica, 116, 148-164.

Denny-Brown, D., and Pennybacker, J. (1938). Fibrillation and fasciculation in voluntary muscle. Brain, 61, 311-334.

Forster, F. M., and Alpers, B. J. (1944). The site of origin of fasciculations in voluntary muscle. Archives of Neurology and Psychiatry (Chicago), 51, 264267.

Forster, F. M., Borkowski, W. J., and Alpers, B. J⿳亠丷厂巾 음 (1946). Effects of denervation on fasciculations in $\rightarrow$ human muscle. Archives of Neurology ando T Psychiatry (Chicago), 56, 276-283.

Gutmann, E., and Hanzlikova, V. (1972). Age Change in the Neuromuscular System. Scientechnicas Bristol.

Hayward, M. (1977). Automatic analysis of the electromyogram in healthy subjects of different ages. Journal of the Neurological Sciences, 33, 397-413.

Hayward, M., and Willison, R. G. (1977). Automatic analysis of the electromyogram in patients with chronic partial denervation. Journal of the Neurological Sciences, 33, 415-423.

Kayser-Gatchalian, M. C. (1973). Late muscular atrophy after poliomyelitis. European Neurology, 10, 371-380.

Milner-Brown, H. S., Stein, R. B., and Yemm, R. (1973). The orderly recruitment of human motor units during voluntary isometric contraction. Journal of Physiology, 230, 359-370. 